\title{
Quantum phase transitions of polar molecules in bilayer systems
}

\author{
Daw-Wei Wang \\ Physics Department, National Tsing-Hua University, Hsinchu, Taiwan, ROC
}

(Dated: August 7, 2021)

\begin{abstract}
We investigate the quantum phase transitions of bosonic polar molecules in a two-dimensional double layer system. We show that an interlayer bound state of dipoles (dimers) can be formed when the dipole strength is above a critical value, leading to a zero energy resonance in the interlayer $s$ wave scattering channel. In the positive detuning side of the resonance, the strong repulsive interlayer pseudo-potential can drive the system into a maximally entangled state, where the wavefunction is a superposition of two states that have all molecules in one of the two layers and none in the other. We critically discuss how the zero-energy resonance, dimer states and the maximally entangled state can be measured in time-of-flight experiments.
\end{abstract}

Introduction: Systems of ultracold atoms have become one of the most promising systems to observe strong correlation effects in many-body physics. Recent progress in the trapping and cooling of chromium atoms [1] and polar molecules [2] further opens new directions for investigating quantum many-body states resulting from the anisotropic dipole-dipole interaction [3]. The long range nature of dipole interaction also makes it possible to study physics in spatially separated multi-component systems, which have been extensively studied in several important subfields of solid state physics: for example, condensation of excitons in bilayer quantum well system [4], interlayer ferromagnetism in bilayer quantum Hall systems [5] and Coulomb drag in coupled quantum wires [6] etc. Therefore it is interesting to investigate what new physics one may expect in the similar systems of polar molecules. Recent example is the proposed chaining phenomena for molecules in a stack of $2 \mathrm{D}$ traps 7], which resembles particle aggregation in colloidal fluids [8].

In this paper we investigate the quantum phase transitions of cold polar molecules trapped in a $2 \mathrm{D}$ double well potential [9]. The electric dipole moment $(D)$ is aligned perpendicular to the layer $(x-y)$ plane by a DC electric field (see Fig. 1(a)) so that the system properties is controlled by a dimensionless dipole strength, $U_{0} \equiv m D^{2} / \hbar^{2} d$, with $m$ being the molecule mass and $d$ being the layer separation. We find three phases that can be observed in three different regimes of $U_{0}$ : For weak dipole strength, $U_{0} \ll 1$, the ground state is just a coupled superfluid (Fig. 1(a)). When $U_{0}$ is increased to be above a critical value, $U_{0}^{*} \sim 0.71$, molecules in different layers can form interlayer bound states, driving the system to be a superfluid of dimers (Fig. 1(b)). Finally, if the molecules are cooled in the large $U_{0}$ regime and the dipole moment is reduced toward $U_{0}^{*}$ adiabatically from above, we demonstrate that the repulsive interlayer pseudo-potential can drive the system to a maximally entangled state as $U_{0} \rightarrow U_{0}^{* *} \sim 1.4$, breaking a global $U(1)$ symmetry via a second order transition. Such maximally entangled (ME) state is a superposition of two macroscopical states (or called GHZ state [10]) that have all molecules in one layer and none in the other (Fig. 1(c)) [11], and therefore will not have any interference pattern even in a single shot time-of-flight measurement. (a)

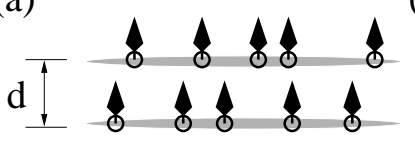

(b)

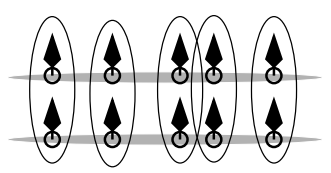

(c)

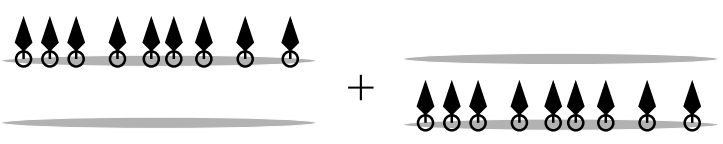

FIG. 1: Three many-body states we consider in this paper: (a) coupled superfluid state, (b) superfluid of dimers, and (c) maximally entangled state.

Pseudo-potential: We start from the low energy scattering properties between two molecules via dipole interaction. In the strong confinement regime, we can first assume only the lowest subband of each layer is occupied and no single particle tunneling between them. The 2D Schrödinger equation in the relative coordinate can then be written to be

$$
-\frac{\hbar^{2}}{m}\left(\partial_{x}^{2}+\partial_{y}^{2}\right) \phi(\mathbf{r})+V_{0 / 1}(\mathbf{r}) \phi(\mathbf{r})=E \phi(\mathbf{r})
$$

where $V_{0 / 1}(\mathbf{r}) \equiv \int d z_{1} d z_{2}\left|\varphi_{0}\left(z_{1}-d / 2\right)\right|^{2}\left|\varphi_{0}\left(z_{2} \mp d / 2\right)\right|^{2}$ $V_{d}\left(\mathbf{r}, z_{1}-z_{2}\right)$ is the bare interaction for the two molecules in the same/different layers. $V_{d}(\mathbf{r}, z)=$ $D^{2}\left(\mathbf{r}^{2}-2 z^{2}\right) /\left(\mathbf{r}^{2}+z^{2}\right)^{5 / 2}$ is the dipole interaction with $\mathbf{r}$ being the relative coordinate in the $x-y$ plane. $\varphi_{0}(z)$ is the lowest confined wavefunction and can be approximated by a Gaussian wavefunction of width $W(W \ll d)$. When finite interlayer tunneling $(t)$ is considered, one has to diagonalize the full two-particle two-layer Hamiltonian. Its effect to Eq. (1) can be shown to be the order of $t^{2} /\left(D^{2} / W^{3}\right)$, and hence negligible in the strong confinement regime as we considered here.

Now, using the standard scattering theory [12], we can derive the following $2 \mathrm{D}$ pseudo-potential:

$$
\mathcal{V}_{\mathrm{ps}}^{(0) /(1)}(\mathbf{r})=-\frac{4 \hbar^{2}}{m} \tan \delta_{0}^{(0) /(1)}(k) \cdot \delta(\mathbf{r})
$$

which reproduces the same $s$-wave phase shift $\left(\delta_{0}^{(0) /(1)}(k)\right)$ as the bare interaction, $V_{0 / 1}(\mathbf{r})$, in large 


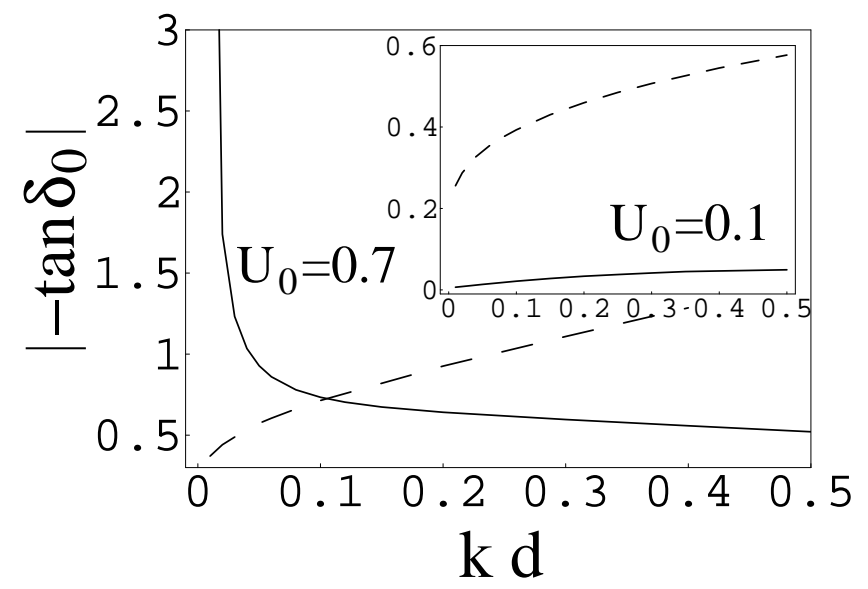

FIG. 2: Intralayer (dashed lines) and interlayer (solid lines) scattering phase shift in the $s$-wave channel as a function of momentum $k d$ near resonance $\left(U_{0}=0.7\right)$. The layer width, $W=0.1 d$, is set much smaller than the interlayer distance $d$. Note that phase shift $\left(\delta_{0}\right)$ of the interlayer scattering is positive, and its sign is reversed for the convenience of comparison with the intralayer results. Inset: Results for $U_{0}=0.1$.

distance. $k$ is the magnitude of the incoming relative momentum. We can show that contributions from higher angular momentum channels can be safely neglected since the typical length scale of dipole interaction, $m D^{2} / \hbar^{2}(\sim 1.5 \mu \mathrm{m}$ for $D \sim 1$ Debye and $m \sim 100$ a.m.u. $)$ is much smaller than the typical condensate size.

Zero energy resonance: In Fig. 2, we show the calculated $s$-wave phase shift as a function of $k d$ for $U_{0}=0.7$ and $U_{0}=0.1$ (inset). When the dipole strength is weak(inset), the phase shift of the interlayer scattering is always much smaller than that of intralayer one as expected, but it becomes much larger when $U_{0}$ is larger. In Fig. 3, we show the numerically calculated low energy $(k d \rightarrow 0) s$-wave phase shift as a function of $U_{0}$, and find a resonance at $U_{0}=U_{0}^{*} \sim 0.71$. Similarly to the Feshbach resonance in typical cold atom systems [13], this zero energy resonance is due to the formation of an interlayer bound state (dimer). Interaction between two dimers can be obtained by integrating out the dimer wavefunction (not shown here). The system ground state for $U_{0}>U_{0}^{*}$ is a superfluid of dimers with a finite binding energy (about $0.1 \times \hbar^{2} / m d^{2}$ at $U_{0}=1$ ), but the actual phase transition position might be shifted from $U_{0}^{*}$ due to the interaction between dimers. We also note that the quantum phase transition from a coupled superfluid to dimer superfluid near $U_{0}^{*}$ is belong to the Ising type transition, because the dimer superfluid phase just breaks a $U(1) / Z_{2}$ symmetry, similar to bosnoic systems near Feshbach resonance as discussed in Ref. [14]. We point that these result cannot be reproduced even qualitatively within the Born approximation in the literature [15], which is valid only when the dipole strength is very weak $\left(U_{0} \ll 1\right)$.

Condensate size near resonance: It is interesting to

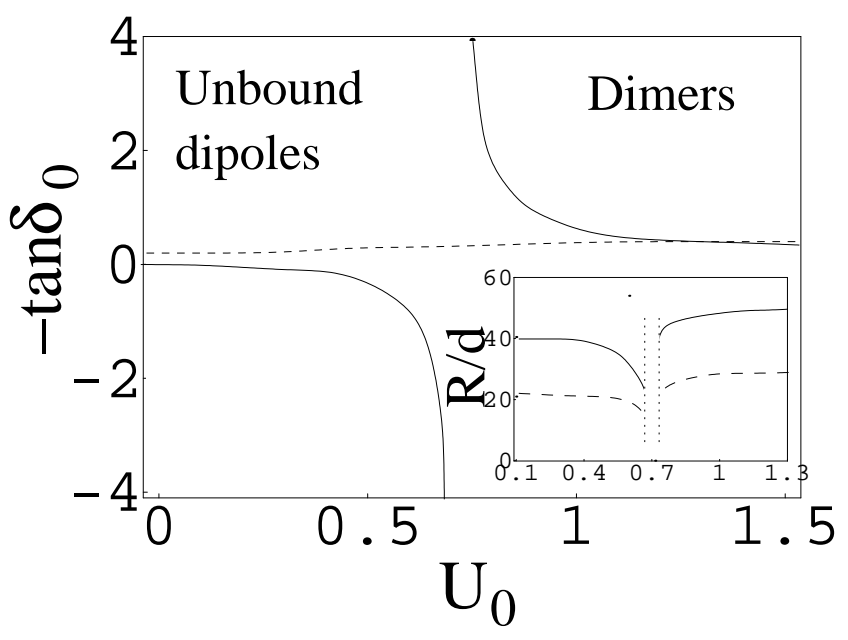

FIG. 3: Zero-energy resonance of the interlayer(solid line) scattering phase shift as a function of $U_{0}$. Dashed line is for the intralayer scattering. Inset: Calculated condensate radius as a function of $U_{0}$. Solid and dashed lines are for harmonic oscillator length, $a_{\mathrm{ho}} \equiv \sqrt{\hbar / m \omega_{\|}}=10 d$, and $5 d$ respectively. Number of molecules in each layer is $N=10^{5}$ and other parameters are the same as in Fig. 2, Our meanfield treatment of Eq. (3) may fails near resonance and therefore we eliminate the data between the two dotted lines.

study how the condensate size is changed when the dipole strength $U_{0}$ is tuned across the resonance point. Using a Gaussian variational wavefunction [16], $\Psi_{ \pm}(\mathbf{r})=$ $\frac{\sqrt{N}}{\sqrt{\pi} R} e^{-|\mathbf{r}|^{2} / 2 R^{2}} \varphi_{0}(z \mp d / 2)$, for the condensate wavefunction in the upper(+) and the lower(-) layers, the radius $R$ in the negative detuning side is then obtained by minimizing the following meanfield energy:

$$
\frac{E}{N}=-t+\frac{\hbar^{2}}{m R^{2}}+m \omega_{\|}^{2} R^{2}+\frac{N \hbar^{2}}{8 \pi m R^{2}} \sum_{i=0,1} A_{0}^{(i)}(R)(3)
$$

Here $\omega_{\|}$is the in-plane trapping frequency, and $A_{0}^{(0) /(1)}(R) \equiv-\int_{0}^{\infty} x \tan \delta_{0}^{(0) /(1)}(x / R) e^{-x^{2} / 4}$ are the dimensionless interaction energies. $t$ is single particle tunneling amplitude and here contributes to a constant only within meanfield approximation. We can also apply a similar method to describe the condensate size of dimers in the positive detuning side $\left(U_{0}>U_{0}^{*}\right)$. When $U_{0}$ is well above $U_{0}^{*}$ (i.e. large binding energy of dimers), the low energy scattering does not break a dimer and the phase sift can be calculated from the interaction between dimers after taking into account their bound state wavefunction [17]. In the inset of Fig. 3, we show the calculated condensate radius as a function of $U_{0}$. In the negative detuning side, the condensate size decreases gradually as $U_{0}$ approaching $U_{0}^{*}$ from below due to the increasing attractive interlayer pseudo-potential (main plot). On the other (positive detuning) side, the size of the dimer condensate grows rapidly due to the repulsive interaction between dimers. Although the meanfield calculation may not be reliable when very close to the 
resonance regime due to the strong momentum dependence of interlayer pseudo-potential, it is reasonable to expect that the sharp shrinking of condensate size near $U_{0}^{*}$ is still qualitatively true. Therefore measuring the dramatical change of condensate size near resonance can provide a clear evidence of zero energy resonance as well as the dimer state in the bilayer system.

Maximally entangled state: In the previous discussion we concentrated on the situation where the dipole strength $U_{0}$ is initially small and adiabatically increased to be above $U_{0}^{*}$. However, in a realistic experiment, the electric dipole moment can be so strong that molecules are cooled directly in the large $U_{0}$ regime with very small transition rate to the dimer state. It is therefore interesting to study how the many-body metastable state is changed when the dipole strength $U_{0}$ is adiabatically tuned toward the critical value $\left(U_{0}^{*}\right)$ from above. From Fig. 3 one can see that there are two regions of interest in this positive detuning side: one is for $U_{0}>U_{0}^{* *} \sim 1.4$ where the effective interlayer interaction is repulsive but still smaller than the intralayer interaction in the long wavelength limit, and the other is for $U_{0}^{*}<U_{0}<U_{0}^{* *}$ where the interlayer pseudo-potential is larger than the intralayer one. Since both inter- and intra-layer interactions are repulsive for $U_{0}>U_{0}^{*}$, hereafter we may neglect the in-plane trapping potential and consider a homogeneous system for simplicity. Assuming all dipoles are in the zero momentum state at zero temperature, we can write the following effective Hamiltonian

$$
\begin{aligned}
H & =-t\left(\hat{a}_{0}^{\dagger} \hat{b}_{0}+\hat{b}_{0}^{\dagger} \hat{a}_{0}\right)+\frac{g_{0}}{2 N}\left[\hat{n}_{a}^{2}+\hat{n}_{b}^{2}\right]+\frac{g_{1}}{N} \hat{n}_{a} \hat{n}_{b} \\
& =\frac{g_{0}}{2 N}(2 N)^{2}-t\left(\hat{a}_{0}^{\dagger} \hat{b}_{0}+\hat{b}_{0}^{\dagger} \hat{a}_{0}\right)+\frac{\Delta g}{N} \hat{n}_{a} \hat{n}_{b}
\end{aligned}
$$

where $\hat{a}_{0}^{\dagger}\left(\hat{b}_{0}^{\dagger}\right)$ are boson creation operators in the upper/lower layer at $k=0$ with $\hat{n}_{a}$ and $\hat{n}_{b}$ being their number operators. $g_{i} \equiv \frac{-4 \hbar^{2} N}{m \Omega} \tan \delta_{0}^{(i)}(k \rightarrow 0)$ is the intra$(i=0)$ or inter- $(i=1)$ meanfield energy with $\Omega$ being the condensate area. $\Delta g \equiv g_{1}-g_{0}<0$ for $U_{0}>U_{0}^{* *}$ and $\Delta g>0$ for $U_{0}^{*}<U_{0}<U_{0}^{* *}$. In the second line of Eq. (4) we have used $\hat{n}_{a}+\hat{n}_{b}=2 N$ as the conserved total number of molecules. We note that although Eq. (4) looks similar to the two-site Bose-Hubbard model with inter-site interaction, the physics described by Eq. (4) is different from the well-known superfluid to Mott-insulator phase transition [18]. For example, the lowest excitation state of our system is always the in-plane gapless phonon mode and therefore no charge gap or commensurate filling are expected even when $t$ is reduced to zero.

Before showing the calculation results, it is instructive to discuss the analytic solutions in three different limits: Firstly, in the limit of $t \ll|\Delta g|$ and $\Delta g<0$, the ground state wavefunction is very close to a Fock state: $\left|\Psi_{\text {Fock }}\right\rangle=\frac{1}{N !} \hat{a}_{0}^{\dagger N} \hat{b}_{0}^{\dagger N}|0\rangle$, with almost zero interlayer phase correlation (or the phase stiffness is very weak). Secondly, for $\Delta g=0$ but with finite tunneling, the ground state is a condensed symmetric coher-

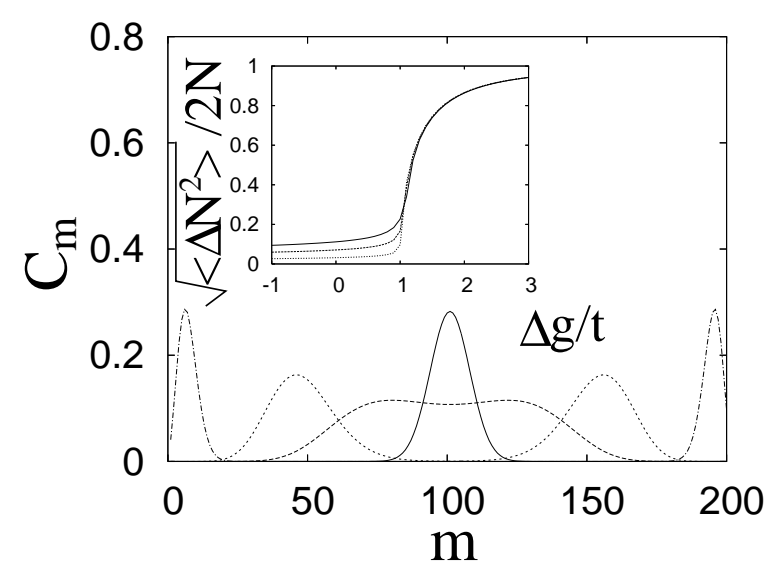

FIG. 4: Many-body wavefunction, $C_{m}$, for total number of $2 N=200$ dipoles. Solid, dashed, dotted, and dash-dotted lines are for $\Delta g / N t=-3,1.05,1.2$, and 3 respectively. Inset: particle number variation as a function of $\Delta g / t$. solid, dashed, and dotted lines are for dipole number $N=40,100$, and 500 in each layer.

ent state, $\left|\Psi_{\text {Sym }}\right\rangle=\frac{1}{2^{N} \sqrt{(2 N) !}}\left(\hat{a}_{0}^{\dagger}+\hat{b}_{0}^{\dagger}\right)^{2 N}|0\rangle$. The two condensate are now phase locked by the single particle tunneling so that there will be a true phase correlation, which can be measured in series of time-of-flight experiments. Finally, in the limit of $\Delta g \gg t>0$, the total energy is minimized by $\left\langle\hat{n}_{a} \hat{n}_{b}\right\rangle=0$, i.e. all dipoles are in one of the two layers and none in the other. The most general ground state wavefunction is a superposition of two macroscopic states, $\left|\Psi_{\mathrm{ME}}\right\rangle=$ $\frac{1}{\sqrt{(2 N) !}}\left(\cos \xi a_{0}^{\dagger 2 N}+\sin \xi e^{i \chi} b_{0}^{\dagger 2 N}\right)|0\rangle$, with tilted angle $\xi$ and phase $\chi$ being arbitrary. Such state is also known as a kind of Greenberg-Horne-Zeilinger state [10], which maximizes the entanglement in many measures. Note that the maximally entangled (ME) state we consider here is spontaneously generated as an exact eigenstate of the system, stabilized by the many-body effects.

The Hamiltonian of Eq. (41) can be easily diagonalized in the Fock states basis: $\left|\phi_{m}\right\rangle \equiv(m !(2 N-m) !)^{-1 / 2} a_{0}^{\dagger m} b_{0}^{\dagger 2 N-m}|0\rangle, \quad$ where $m=0,1, \cdots, 2 N$ is the number of dipoles in the upper layer. The ground state wavefunction can therefore be written to be $\left|\Psi_{G}\right\rangle=\sum_{m=0}^{2 N} C_{m}\left|\phi_{m}\right\rangle$ with $\left\{C_{m}\right\}$ being the eigenvector associated with the lowest eigenenergy of Hamiltonian matrix, $\left\langle\phi_{m}|H| \phi_{n}\right\rangle$. Similar approach can also be applied to systems of finite trapping potential. In Fig. 4 we show the exact numerical results of the ground state wavefunction $\left(C_{m}\right)$ for different values of $\Delta g / t$. One can see that for smaller $\Delta g / t$ (solid line), the wavefunction is peaked at $m=N$ with a finite distribution width to gain tunneling energy. When $\Delta g / t$ is close to one, the single peak distribution becomes unstable and tends toward a double-peak distribution. Increasing $\Delta g$ further (i.e. reducing $U_{0}$ in the positive detuning 
side) drives the distribution to peak near $m=0$ and $2 N$, indicating an ME state as discussed above. In the inset of Fig. 4, we show the particle number variation of the ground state, $\left\langle\Delta N^{2}\right\rangle \equiv\left\langle\psi_{G}\left|\left(a_{0}^{\dagger} a_{0}-b_{0}^{\dagger} b_{0}\right)^{2}\right| \Psi_{G}\right\rangle$, as a function of $\Delta g / t$ for various numbers of dipoles per layer $(N)$. One can see that in the thermodynamic limit (i.e. keeping $\Delta g \propto N / \Omega$ fixed as $N \rightarrow \infty$ ), there is a sharp phase transition exactly at $\Delta g / t=1$, above which $\sqrt{\left\langle\Delta \hat{N}^{2}\right\rangle} / 2 N$ becomes finite and eventually saturates.

To understand such a sharp phase transition from a coherent state to the ME state, we can rewrite Eq. (4) as a spin model (upto a constant) [11]: $H=-2 t \hat{S}_{x}-\frac{\Delta g}{N} \hat{S}_{z}^{2}$, where $\hat{S}_{x} \equiv \frac{1}{2}\left(a_{0}^{\dagger} b_{0}+b_{0}^{\dagger} a_{0}\right), \quad \hat{S}_{y} \equiv \frac{i}{2}\left(b_{0}^{\dagger} a_{0}-a_{0}^{\dagger} b_{0}\right)$, and $\hat{S}_{z} \equiv \frac{1}{2}\left(a_{0}^{\dagger} a_{0}-b_{0}^{\dagger} b_{0}\right)$. The total spin is then given by $\hat{\mathbf{S}}^{2}=\frac{1}{4}\left(\hat{n}_{a}+\hat{n}_{b}\right)\left(\hat{n}_{a}+\hat{n}_{b}+2\right)$. In the thermodynamic limit $(N \rightarrow \infty)$, we can treat $\mathbf{S}$ a classical spin: $\mathbf{S}=N(\sin \theta \cos \phi, \sin \theta \sin \phi, \cos \theta)$ with $\theta$ and $\phi$ being the spin angles in 3D space. Therefore the ground state is obtained by minimizing the energy $E(\theta, \phi) / N=-2 t \sin \theta \cos \phi-\Delta g \cos ^{2} \theta$ with respect to $\theta$ and $\phi$. Since $\phi$ must be zero to gain the tunneling energy, we can expand $E(\theta, 0)$ to the leading order of $\eta=\theta-\pi / 2$ and obtain a Ginsberg-Landau type energy: $E(\eta, 0) / N=-2 t+(t-\Delta g) \eta^{2}+\frac{1}{3}(\Delta g-t / 4) \eta^{4}$, which shows a clear second order phase transition at $\Delta g=t$. The variation of particle number (i.e. variation of $\hat{S}_{z}$ ) scales as $(\Delta g-t)^{1 / 2}$ near the transition point.

Before concluding, we remark on several experimental issues for observing the maximally entangled state in the bilayer system. First, for a typical polar molecule $U_{0}$ can be as large as $4-5$ and can be easily reduced to zero by decreasing the external DC electric field. Secondly we can show that phase separation (i.e. dipoles accumulate inhomogeneously in layers) is unlikely to occur because it causes extra kinetic energy compare to the homogeneously entangled state. Thirdly, the three-body collisions induced transition to dimer states can be strongly suppressed in the ME state, because most molecules now are in one of the layers and very few molecules are in the other layer. As a result the ME state we proposed here should be a long-lived meta-stable state and hence can be easily observed in experiments. Finally, unlike the interference pattern of two independent condensates [19], the fringe contrast of such entangled state will disappear even in a single-shot time-of-flight measurement as $U_{0}$ is adiabatically tuned to be lower than $U_{0}^{* *}$ from above. This is because the maximally entangled state, $\left|\Psi_{M F}\right\rangle$, is a superposition of two macroscopic states, and hence very fragile to collapse in any quantum measurement. Therefore the disappearance of interference pattern in the positive detuning side could be a direct experimental evidence of such maximally entangled state.

In summary, we demonstrate that loading polar molecules into a bilayer system can result in several interesting new physics, including zero energy resonance, interlayer bound states (dimers), and a second order quantum phase transition toward a maximally entangled state. These new phenomena should be easily observable using present experimental techniques.

This work is supported by NSC (Taiwan).
1 J. Stuhler et al., Phys. Rev. Lett. 95, 150406 (2005).

2 J. Doyle, Eur. Phys. J. D 31, 149 (2004); J.D. Weinstein et al., Nature 395, 148 (1998); H.L. Bethlem et al., Nature 406, 491 (2000); A.J. Kerman et al., Phys. Rev. Lett. 92, 033004 (2004).

${ }^{3}$ K. Góral et al., Phys. Rev. Lett. 88, 170406 (2002); R. Barnett, et. al., Phys. Rev. Lett. 96, 190401 (2006); A. Micheli, Nature Phys. 2,341 (2006); H.P. Büchler, et al., cond-mat/0607294.

4 X. Zhu, et al., Phys. Rev. Lett. 74, 1633 (1995); For a recent review, see L. V. Butov, Solid State Commun. 127, 89 (2003), and reference therein.

${ }^{5}$ For a review, see J.P. Eisenstein, S.M. Girvin and A.H. MacDonald, in Perspectives in Quantum Hall Effects edited by S. Das Sarma and A. Pinczuk (John Wiley \& Sons, New York, 1997), and reference therein.

6 P. Debray, et al., J. Condens. Matter 13, 3389 (2001); R. Klesse and A. Stern, Phys. Rev. B 62, 16912201316925 (2000); M. Yamamoto, et al., Science 313, 204 (2006).

7 D.-W. Wang, et al., Phys. Rev. Lett. 97, 180413 (2006).

8 P.G. De Gennes and P.A. Pinous, Phys. kondens. Materie 11, 189 (1970); P.I.C. Teixeira, et al., J. Phys. Condens. Matter 12, R411 (2000).

9 Z. Hadzibabic, et al., Nature, 4411118 (2006); Z. Hadzibabic, et al., Phys. Rev. Lett. 93180403 (2004).

10 D.M. Greenberger, et al., Am. J. Phys. 58, 1131 (1990);
K. Mølmer and A. Sørensen, Phys. Rev. Lett. 82, 1835 (1999); L. You, Phys. Rev. Lett. 90, 030402 (2003).

11 C. Monroe, et. al., Science 272, 1131 (1996); J.I. Cirac, et al., Phys. Rev. A, 57, 1208 (1998); D. Gordon and C.M. Savage, ibid., 59, 4623 (1999); A. Micheli, et al., ibid., 67, 013607 (2003).

12 K. Huang and C.N. Yang, Phys. Rev. 105, 767 (1957); B.J. Verhaar, et al., J. Phys. A 17595 (1984); A. Derevianko, Phys. Rev. A 67, 033607 (2003).

13 M.W. Zwierlein, et al., Phys. Rev. Lett. 92, 120403 (2004); M.W. Zwierlein, Nature 435 , 1047 (2005).

14 M.W.J. Romans, et al., Phys. Rev. Lett. 93, 020405 (2004).

15 L. Santos et al., Phys. Rev. Lett. 85, 1791 (2000); L. Santos et al., Phys. Rev. Lett.90, 250403 (2003); Duncan H. J. O'Dell et al., Phys. Rev. Lett. 92, 250401 (2004).

16 S. Yi and L. You, Phys. Rev. Lett. 92, 193201 (2004).

17 D.-W. Wang, unpublished.

18 D. Jaksch, et al., Phys. Rev. Lett. 81, 3108 (1998); M Greiner, et al., Nature 415, 39 (2002); M. Albiez, et al., Phys. Rev. Lett. 95, 010402 (2005).

19 J. Javanainen and S.M. Yoo, Phys. Rev. Lett., 76, 161 (1996); Y. Castin and J. Dalibard, Phys. Rev. A, 55, 4330 (1997); M.R. Andrews, et al., Science 275, 637 (1997). 